\title{
ESTRESSE LABORAL: ANÁLISE DA PRODUÇÃO CIENTÍFICA BRASILEIRA NA SCIELO E BVS-PSI
}

\author{
Ana Paula Porto Noronha \\ Dario Cecilio Fernandes $\star \star$
}

\begin{abstract}
RESUMO
O presente estudo teve como objetivo a análise da produção de artigos relacionada ao construto estresse. Para tanto, procedeu-se a uma busca em bases de dados eletrônicas (SciELO e BVS-Psi), utilizando-se das palavraschave estresse, estresse laboral, estresse ocupacional e testes psicológicos. Foram identificados 96 artigos, dos quais se analisaram: ano de publicação, sexo do autor, revistas publicadas e instrumentos utilizados. Os resumos em sua grande maioria se referem a revistas de psicologia, entretanto, foram encontrados artigos em revistas de Educação, Medicina, Enfermagem, dentre outras. Os resultados indicaram que a maior parte dos resumos era referente a pesquisas, sendo que o principal tema era o levantamento sintomatológico. Em relação a publicações se observa uma maior concentração em Estudos de Psicologia (Campinas) e Psicologia Reflexão e Crítica. Além disso, nota-se um grande aumento na produção científica a partir do ano de 1999.
\end{abstract}

Palavras-chave: avaliação psicológica; testes psicológicos; construção de testes.

\section{OCCUPATIONAL STRESS: ANALYSIS OF THE SCIENTIFIC PRODUCTION IN ScIELO AND BVS-PSI}

\begin{abstract}
The present study had the objective of analysis the article production related to stress. For this such a way, it was proceeded a search in electronic databases (SciELO and BVS-Psi), using the keywords stress, labor stress, occupational stress and psychological assessments. 96 articles were selected, and were analyzed by the year of publication, sex of the author, theoretical review and the used instruments. The summaries majority was relate to psychology magazines, however, had been found articles in magazines of Education, Medicine, Nursing,

^ Doutora em Psicologia: Ciência e Profissão pela Pontifícia Universidade Católica de Campinas; docente do Programa de Pós-Gradução Stricto Sensu em Psicologia da Universidade São Francisco; bolsista de Produtividade em Pesquisa do CNPq. Endereço: Universidade São Francisco, Faculdade de Filosofia Ciências e Letras. Avenida Senador Lacerda Franco, sn. CEP: 13251-900 - Itatiba, SP - Brasil.

E-mail: ana.noronha@saofrancisco.edu.br

* Acadêmico do Curso de Psicologia da Universidade São Francisco, bolsista de Iniciação Científica - FAPESP.
\end{abstract}


amongst others. The results had indicated that most of the summaries were referring to the research, being that the main subject was the symptoms survey. In relation to the publication were found a concentration in Estudos de Psicologia (Campinas) and Psicologia Reflexão e Crítica. Besides that, were noticed a increase in the scientific production above the year of 1999.

Keywords: psychological assessment; psychological tests; tests construction.

\section{INTRODUÇão}

A avaliação da produção científica é uma prática indispensável à ciência, já que ela permite identificar, com maior rigor, os assuntos freqüentemente tratados, os tipos de estudos realizados, dentre outros aspectos não menos relevantes. Ainda, ao diagnosticar o "estado da arte" de determinada área de conhecimento, têm-se em conseqüência, os indicadores, que tal como pontuado por Mugnaini, Carvalho e Campanatti-Ostiz (2006), ilustram a amplitude da ciência produzida no país.

Nesse sentido, a magnitude obtida por meio da análise da produção tende a oferecer novas perspectivas para pesquisas futuras, partindo do que já foi desenvolvido até então. Se assim os autores o fizessem, ou seja, se a realização de novas pesquisas tivesse como suporte o universo estudado, o desenvolvimento da ciência seria mais organizado (MEIS; LETA, 1996).

A avaliação da produção científica, para Witter $(1979,2006)$, faz-se em contextos distintos e, para tanto, recorre-se a instrumentos variados de coleta da informação, com características mais simples ou complexas. De acordo com a autora, a observação do que já foi produzido deve ser constante em todas as ciências, embora as metodologias empregadas possam variar. Dentre as maiores relevâncias, encontram-se o tipo de autoria, o tipo de estudo (se teórico ou de pesquisa), o tema investigado, o delineamento do trabalho (no caso das pesquisas), os instrumentos utilizados, e outras informações.

O presente estudo versa sobre a análise da produção científica sobre o estresse. O termo provém do inglês stress, e teve sua origem vinculada à área biológica. Cannon e Selye foram os precursores que estudaram as respostas fisiológicas apresentadas por animais quando expostos a situações aversivas ou ameaçadoras (CARLSON, 1995). Cannon foi o responsável pela criação do termo homeostase, que significa manter o organismo em um estado de equilíbrio interno (SELYE, 1982), sendo que o conceito colaborou para o avanço dos estudos sobre estresse. Em acréscimo, as fontes estressoras são consideradas como aquelas que têm o efeito de interferir na dinâmica da vida do organismo (HINKLE, 1987).

Autores, incluindo-se os citados anteriormente, dedicaram-se com afinco à investigação do fenômeno. Selye $(1956,1982)$ acreditava que o estresse podia ser entendido como o resultado de um estímulo maior do que o corpo possa suportar, provocando conseqüências cognitivas e somáticas. Na mesma direção, Haan (1982) se referia ao estresse como uma situação que causa pressão e desconforto ao indivíduo, e que normalmente ocorre diante da necessidade do ser humano se 
adaptar às mudanças. Ao lado disso, Holroyd e Lazarus (1982) destacaram que, se as demandas internas e externas forem maiores que os recursos individuais para lidar com elas, ocorre, então, o estresse psicológico.

Embora o construto venha sendo amplamente estudado, não há consenso entre os autores quanto à melhor definição. O desacordo entre eles, para Pearlin (1982), centra-se na dificuldade de determinar "onde" e "como" as respostas estressoras podem ser identificadas. No entanto, segundo o autor, parece haver consenso entre alguns teóricos de que o estresse é uma resposta do organismo a algumas situações.

Na sua revisão do conceito de estresse, Hinkle (1987) endossa as concepções de Pearlin (1982), já que acredita que não há uma definição amplamente aceita, e que a definição das ocorrências do construto, ou a melhor forma de medi-lo ainda necessitam de investigações. O autor destaca que algumas definições chegam a ser contraditórias, embora haja maior concordância entre os pesquisadores quanto às fontes de estresse.

No que se refere, mais especialmente, aos estímulos potencialmente estressores, Mills (1985) os dividia em dois grandes grupos, a saber, os físicos (exercícios físicos, por exemplo) e psicológicos (situações caracterizadas pela novidade, antecipação ou imprevisibilidade de eventos, mudanças). Além disso, a categorização determinava se a origem do estímulo era externa ao organismo (acidentes, mudanças de chefia e morte de um ente querido, por exemplo) ou interna (características pessoais como ansiedade, timidez, crenças irracionais, dentre outras).

Conforme Carlson (1995) e Hamilton (1982) existem estímulos que terão a resposta de estresse vinculada à capacidade física e ao estado geral de saúde do organismo como, por exemplo, temperaturas extremas, privações diversas (alimento, água, sono, social), traumas físicos, entre outros, sendo que, dentro de uma estreita faixa de variabilidade, provocam respostas de estresse em praticamente todos os organismos a eles expostos. Tais estressores são chamados também de "biogênicos", por estarem relacionados à sobrevivência do organismo.

Os sintomas do estresse, por sua vez, foram discutidos em trabalhos diversos. Nesse particular, Maes, Vingerhorts e Van Heck (1987) consideram estressores eventos que sobrecarregam ou excedam os recursos adaptativos da pessoa. Essa falta de recurso para o organismo lidar com algo novo ou a ele se adaptar tende a gerar os sintomas.

Podem-se considerar, de acordo com Mota, Franco e Motta (1999), duas classes principais de aspectos associadas ao risco de natureza psicológica, como os estímulos considerados estressantes e os significados que os sujeitos conferem às mudanças e aos eventos. Em uma perspectiva diferente, Lipp (2000) concebe que a reação ao estresse pode ser dividida em quatro aspectos, quais sejam, alerta, resistência, quase-exautão e exaustão.

A primeira fase é quando o sujeito é exposto a uma situação geradora de tensão, então, ele se prepara para a ação, ocasionada por diversas alterações bioquímicas. Quando o agente estressor não é eliminado, o sujeito passa para a 
fase de resistência, quando o sujeito utiliza recursos para se reequilibrar (energia adaptativa). Assim, quando isso ocorre há uma sensação de que o problema foi resolvido, entretanto, há um aumento no cansaço excessivo, sendo que o sujeito não o consegue identificar. No estágio três, o organismo está tão enfraquecido que não consegue resistir e/ou se adaptar, de tal modo que aparecem as doenças. Quando o fator ou situação estressante, sendo uma ou várias, permanece por muito tempo, então, o sujeito passa para a fase de exaustão. Nesse estágio observa-se a exaustão física e psicológica, o que aumenta a probabilidade do aparecimento de doenças, sejam elas físicas ou psicológicas.

Não é difícil supor que o estresse atinge áreas diversas da vida do sujeito, tal como a profissional, independentemente da idade. Em relação ao último aspecto, por exemplo, Lipp, Arantes e Buriti (2002) estudaram o estresse em crianças e concluíram que aquelas que estão em idade escolar estão expostas a ele, o que é ocasionado por adaptações geradas pelo processo natural de desenvolvimento, embora nem todas desenvolvam os sintomas. Dentre os estudos realizados com adultos, destaque pode ser dado aos que abordam a relação entre trabalho e estresse, sendo que podem ser justificados ao se levarem em consideração os efeitos prejudiciais encontrados em ambientes institucionais.

Já o estresse ocupacional tem sido objeto de análise de vários autores (CADIZ et al., 1997; JAFFE, 1995), gerando numerosas investigações e transcendendo o campo designado pela Psicologia Organizacional e do Trabalho. Segundo Beehr (1998), o estresse deveria ser tratado não apenas como uma variável, mas como diversas variáveis relacionadas aos estímulos do ambiente de trabalho.

No cotidiano, a palavra estresse é muito usada como algo que é desencadeado por um acontecimento ruim, entretanto, Calais, Andrade e Lipp (2003) afirmam que o aparecimento do estresse não ocorre apenas em situações adversas, já que é possível compreendê-lo como uma reação intensa do organismo diante de um evento bom ou mau que altera o cotidiano do sujeito. Outro equívoco presente nas concepções mais contemporâneas sobre estresse é a associação do construto mais fortemente vinculada às condições de vida do homem do século XX. Em que pesem tais considerações, Selye (1982) afirma que nossos antepassados já vivenciavam situações estressantes.

Apesar de o estudo dos eventos estressores contar com um considerável desenvolvimento histórico, a maior parte da literatura empiricamente validada surgiu somente nas últimas duas décadas (BRIERE, 1997), o que abona a necessidade de estudos na área. Ainda no que se refere à justificativa do presente estudo, convém destacar que parece pertinente que a produção da área seja avaliada, a fim de permitir identificar os avanços e as necessidades. Isto posto, o presente estudo destinou-se a investigar duas bases de dados utilizando como critério para a consulta os unitermos estresse, estresse laboral, estresse ocupacional e testes psicológicos. 


\section{MÉTOdo}

\section{Fonte e procedimento}

As fontes utilizadas para a presente pesquisa foram as bases de dados SciELO e Biblioteca Virtual da Saúde (BVS-Psi) por serem consideradas as mais completas para o acesso à produção nacional. A consulta se deu por meio das palavras-chave estresse, estresse laboral, estresse ocupacional e testes psicológicos. Ressalta-se que os trabalhos acessados datam do período entre 1984 e 2005, por se tratar do período disponível pelas bases de dados.

Foram encontrados 96 resumos de artigos, os quais foram analisados com a utilização de informações advindas da autoria e do conteúdo do resumo. Em "autoria" identificou-se o tipo (individual ou múltipla) e o gênero dos autores. No "conteúdo" investigou-se o tema tratado no estudo. Também foram avaliados: ano de publicação, revistas publicadas e se houve utilização de instrumentos.

Para realizar a análise de conteúdo, foram criadas categorias pelos pesquisadores, de acordo com os princípios de Bardin (1971), sendo que a inclusão dos dados coletados nas respectivas categorias se deu de maneira consensual. Para a compreensão dos dados obtidos nos resumos dos artigos foram estabelecidas cinco categorias de análise cujas definições vêm a seguir.

Revisão teórica: artigos teóricos que revisavam o construto do estresse.

Comparação com outros construtos: consideraram-se os artigos de pesquisa nos quais havia dois ou mais construtos em discussão.

Construção e validação de instrumentos: nesse item foram incluídos artigos que tratavam de construção, validação, precisão e padronização de instrumentos relacionados a estresse.

Levantamento de sintomatologia: essa categoria foi composta por manuscritos que apresentavam informações ou levantamento de sintomas relacionados ao construto estudado.

Outros: nessa categoria foram analisados os artigos que não se encaixaram nas categorias anteriores.

\section{Resultados}

Os resultados que se seguem foram extraídos dos resumos consultados. Quanto ao gênero da autoria, tomou-se como referência apenas o primeiro autor, sendo que foi possível observar que $75 \%(\mathrm{~N}=72)$ são do sexo feminino e $25 \%$ $(\mathrm{N}=24)$ do sexo masculino. Ainda no que diz respeito à autoria, investigou-se a quantidade de autor por trabalho, o que revelou uma grande variabilidade. Os artigos variaram de um a dez autores, média de 2,73 autores e $\mathrm{DP}=1,88$. Na Tabela 1, estão dispostas as freqüências e respectivas porcentagens referentes ao número de autores. 
Tabela 1. Freqüência e porcentagem do número de autores

\begin{tabular}{ccc}
\hline Número de autores & Freqüência & Porcentagem \\
Única & 25 & $26,0 \%$ \\
Dois & 33 & $34,4 \%$ \\
Três & 16 & $16,7 \%$ \\
Quatro & 7 & $7,3 \%$ \\
Cinco & 7 & $7,3 \%$ \\
Seis & 1 & $1,0 \%$ \\
Sete & 5 & $5,2 \%$ \\
Nove & 1 & $1,0 \%$ \\
Dez & 1 & $1,0 \%$ \\
Total & 96 & $100,0 \%$ \\
\hline
\end{tabular}

Como já explicitado no método, a fim de se investigar o conteúdo dos trabalhos, foram definidas cinco categorias de análise. Os achados permitiram observar que a maior parte refere-se à pesquisa $(69,8 \%)$, em detrimento de artigos teóricos (30,2\%). Dentre as pesquisas, o Levantamento de Sintomatologia esteve presente em 28,1\% dos resumos, enquanto a categoria Construção e Validação de Instrumentos ficou com 19,8\% e a Comparação com outros Construtos, com $17,7 \%$. Na Tabela 2, observa-se a distribuição dos resumos pelas categorias.

Tabela 2. Freqüência e porcentagem da classificação do conteúdo dos resumos

\begin{tabular}{lcc}
\hline & Freqüência & Porcentagem \\
Revisão teórica & 29 & $30,2 \%$ \\
Comparação outros construtos & 17 & $17,7 \%$ \\
Construção - validação & 19 & $19,8 \%$ \\
Sintomatologia & 27 & $28,1 \%$ \\
Outros & 4 & $4,2 \%$ \\
Total & 96 & $100 \%$ \\
\hline
\end{tabular}

Além disso, quanto ao conteúdo dos resumos, analisou-se o uso de instrumentos de avaliação; nesse sentido, observou-se que 47,9\% (N=46) dos artigos utilizam algum tipo de instrumento para a avaliação do estresse, seja com o objetivo de construir, comparar populações ou instrumentos ou, ainda, realizar levantamentos de sintomas mais freqüentes. Além disso, foi analisada a origem da publicação, cujos dados encontram-se na Tabela 3.

Foram encontrados 36 periódicos com publicações sobre o tema, sendo que a área de proveniência não foi necessariamente a Psicologia, tendo sido encontradas revistas de Educação, Medicina, Enfermagem, dentre outras. A maior concentração de artigos, no entanto, deu-se em revistas de Psicologia, como a Estudos de Psicologia (Campinas) e Psicologia Reflexão e Crítica. Outro aspecto interessante refere-se ao local de publicação dos artigos e, nesse particular, é possível compreender que há uma concentração numa determinada região, mais especialmente na região sudeste do país. 
Tabela 3. Relação dos periódicos com publicações sobre estresse

\begin{tabular}{|c|c|c|c|}
\hline \multicolumn{2}{|c|}{ Revista } & \multirow{2}{*}{$\begin{array}{l}\text { Freqü ência } \\
5\end{array}$} & \multirow{2}{*}{$\begin{array}{c}\text { Porcentagem } \\
5,2 \%\end{array}$} \\
\hline RS & Alethéia & & \\
\hline RJ & Arquivos Brasileiros de Psicologia & 1 & $1,0 \%$ \\
\hline SP & Boletim de Psicologia & 3 & $3,1 \%$ \\
\hline ES & Cadernos de Pesquisa da UFES & 1 & $1,0 \%$ \\
\hline RJ & Ciência \& Saúde Coletiva & 1 & $1,0 \%$ \\
\hline SP & Estudos de Psicologia (Campinas) & 13 & $13,5 \%$ \\
\hline RN & Estudo de Psicologia (Natal) & 4 & $4,2 \%$ \\
\hline RJ & In formação Psiquiátrica & 3 & $3,1 \%$ \\
\hline PR & Interação (Curitiba) & 2 & $2,1 \%$ \\
\hline \multirow[t]{2}{*}{ RJ } & Jornal Brasileiro de Psiquiatria & 7 & $7,3 \%$ \\
\hline & Mudanças & 2 & $2,1 \%$ \\
\hline \multirow[t]{2}{*}{ SP } & Paidéia & 1 & $1,0 \%$ \\
\hline & Pensando Famílias & 1 & $1,0 \%$ \\
\hline RS & Psico (Porto Alegre) & 3 & $3,1 \%$ \\
\hline SP & Psico USF & 5 & $5,2 \%$ \\
\hline PR & Psicologia Argumento & 5 & $5,2 \%$ \\
\hline DF & Psicologia Ciência e Profissão & 2 & $2,1 \%$ \\
\hline PR & Psicologia Corporal & 1 & $1,0 \%$ \\
\hline SP & Psicologia Escolar e Educacional & 2 & $2,1 \%$ \\
\hline PR & Psicologia em Estudo & 5 & $5,2 \%$ \\
\hline RS & Psicologia Reflexão e Crítica & 8 & $8,3 \%$ \\
\hline DF & Psicologia: Teoria e Pesquisa & 5 & $5,2 \%$ \\
\hline SP & Psikhe & 1 & $1,0 \%$ \\
\hline SP & Revista ABP-APAL & 1 & $1,0 \%$ \\
\hline SP & Revista Brasileira de Crescimento e Desenvolvimento Humano & 1 & $1,0 \%$ \\
\hline SP & Revista Brasileira de Pesquisa em Psicologia & 1 & $1,0 \%$ \\
\hline SP & Revista Brasileira de Psicodrama & 1 & $1,0 \%$ \\
\hline SP & Revista Brasileira de Terapia Cognitiva & 1 & $1,0 \%$ \\
\hline MG & Revista da Socie dade Brasileira de Psicologia Hospitalar & 1 & $1,0 \%$ \\
\hline RS & Revista da Sociedade de Psicologia do Rio Grande do Sul & 1 & $1,0 \%$ \\
\hline CE & Revista de Psicologia & 2 & $2,1 \%$ \\
\hline RS & Revista de Psiquiatria do Rio Grande do Sul & 1 & $1,0 \%$ \\
\hline SP & Revista de Saúde Pública & 1 & $1,0 \%$ \\
\hline SP & Revista Latino-Americana de Enfermagem & 2 & $2,1 \%$ \\
\hline SC & Revista Psicologia: organizações e trabalho & 1 & $1,0 \%$ \\
\hline \multirow[t]{2}{*}{ PR } & Teoria e Prática da Educação & 1 & $1,0 \%$ \\
\hline & Total de Artigos & 96 & $100,0 \%$ \\
\hline
\end{tabular}

Por fim, a Tabela 4 procurou relacionar o ano de publicação com o conteúdo do resumo, definido anteriormente. Uma primeira análise permitiu identificar que houve um aumento das publicações ao longo dos períodos. Em contrapartida, o pequeno número de trabalhos na década de 1980 também pode estar associado ao pouco desenvolvimento das revistas eletrônicas à época. Vale destacar que os artigos foram identificados pela Internet, podendo-se inferir que, com a expansão dos sites de buscas, as revistas disponíveis eletronicamente tiveram uma maior visibilidade, estimulando a publicação. Assim, embora os resultados tenham apontado o aumento da publicação, há que se considerar os outros aspectos acima discutidos. 
Tabela 4. Ano de publicação dos resumos e conteúdos abordados

\begin{tabular}{|c|c|c|c|c|c|cc|}
\hline Ano & $\begin{array}{c}\text { Revisão } \\
\text { teórica }\end{array}$ & $\begin{array}{c}\text { Comparação } \\
\text { outros } \\
\text { construtos }\end{array}$ & $\begin{array}{c}\text { Construção } \\
\text { validação }\end{array}$ & Sintomatologia & Outros & Total & $\%$ \\
\hline 1984 & 1 & 0 & 0 & 0 & 0 & 1 & 1,04 \\
1985 & 1 & 0 & 0 & 2 & 0 & 3 & 3,12 \\
\hline 1987 & 1 & 1 & 0 & 0 & 0 & 2 & 2,08 \\
\hline 1989 & 0 & 0 & 0 & 0 & 1 & 1 & 1,04 \\
1990 & 0 & 1 & 0 & 0 & 0 & 1 & 1,04 \\
\hline 1992 & 2 & 0 & 0 & 1 & 0 & 3 & 3,12 \\
1993 & 1 & 0 & 0 & 1 & 0 & 2 & 2,08 \\
\hline 1994 & 1 & 0 & 0 & 0 & 0 & 1 & 1,04 \\
\hline 1995 & 1 & 0 & 0 & 0 & 0 & 1 & 1,04 \\
\hline 1996 & 3 & 1 & 1 & 1 & 0 & 6 & 6,25 \\
\hline 1997 & 3 & 0 & 0 & 0 & 0 & 3 & 3,12 \\
1998 & 2 & 0 & 0 & 5 & 0 & 7 & 7,29 \\
\hline 1999 & 6 & 1 & 1 & 1 & 0 & 9 & 9,37 \\
2000 & 0 & 1 & 2 & 0 & 0 & 3 & 3,12 \\
\hline 2001 & 0 & 1 & 2 & 1 & 0 & 4 & 4,2 \\
\hline 2002 & 3 & 3 & 1 & 8 & 0 & 15 & 15,63 \\
\hline 2003 & 2 & 1 & 2 & 5 & 2 & 12 & 12,5 \\
\hline 2004 & 1 & 3 & 5 & 0 & 1 & 10 & 10,42 \\
\hline 2005 & 1 & 3 & 5 & 3 & 0 & 12 & 12,5 \\
\hline Total & 29 & 16 & 19 & 28 & 4 & 96 & 100,0 \\
\hline
\end{tabular}

No que se refere aos temas trabalhados nos resumos, o levantamento de sintomas recebeu destaque, seguido das revisões teóricas. Já a construção de instrumentos e a comparação com outros construtos foram menos privilegiadas. Ao lado disso, a revisão teórica foi responsável pelos primeiros trabalhos sobre o tema, sendo que outros apareceram apenas mais recentemente, como é o caso dos relacionados à construção de instrumentos.

\section{DiscuSSÃo E CONSIDERAÇõeS FINAIS}

O estresse vem sendo estudado em diversas áreas de conhecimento, já que é possível relacioná-lo a diferentes facetas do homem. Em razão disso, o interesse pela área é vasto, uma vez que tende a afetar o ser humano no seu dia-a-dia, em contextos variados. Essa pesquisa teve como objetivo analisar as publicações sobre estresse nos últimos anos.

Mais especialmente, o objetivo do presente estudo era traçar um sucinto panorama das publicações a respeito de estresse, por meio de resumos obtidos em duas bases de dados, quais sejam, SciELO e Biblioteca Virtual da Saúde (BVSPsi). Trata-se de um estudo de levantamento e, em razão disso, a amplitude da generalização é bastante restrita. Tendo isso em vista, as considerações que seguem têm o intuito de apontar alguns elementos e promover reflexões, e tal como pontuado por Mugnaini, Carvalho e Campanatti-Ostiz (2006), ao diagnosticar o "estado da arte" de determinada área de conhecimento, tem-se em conseqüência os indicadores que ilustram a amplitude da ciência. 
A autoria dos resumos foi predominantemente feminina e o trabalho foi elaborado por mais de um autor, em sua maioria, embora tenham sido encontrados trabalhos de até dez autores. A predominância do gênero feminino pode ser compreendida à luz da profissão (psicologia), já que a maior parte das revistas publicadas refere-se a esta área, na qual a maioria é feminina. Por sua vez, a autoria múltipla (dois ou mais autores) foi referenciada por vários autores, dentre eles Witter $(1979,2006)$. Esse tipo de publicação é desejável, já que tende a promover intercâmbios e reflexões mais consistentes.

Os artigos de pesquisa se sobressaem em detrimento dos teóricos, sendo que, dentre as pesquisas, os levantamentos de sintomatologia aparecem com algum destaque, o que sugere estarem em consonância com os apontamentos de Maes, Vingerhorts e Heck (1987), na direção de que os sintomas do estresse foram discutidos exaustivamente em trabalhos diversos, embora não haja concordância entre as concepções de estresse e dos próprios sintomas (MILLS, 1985; HINKLE, 1987; PEARLIN, 1982).

Há que se ressaltar o grande número de periódicos nos quais foram encontrados manuscritos sobre o tema. Como já destacado, dentre os periódicos, estavam presentes alguns representando áreas diversas; dentro da Psicologia, as duas que publicaram maior número de artigos são revistas tradicionais na área, o que pode justificar o volume encontrado. Ao lado disso, a região sudeste foi a que apresentou mais artigos sobre estresse, muito possivelmente por ser uma região onde há maior concentração de periódicos, assim como de pesquisadores.

Embora, tal como preconizado por Selye (1982), situações estressantes já fossem vivenciadas por antepassados longínquos, o que gerou reflexões e estudos variados, a maior parte da literatura empiricamente validada surgiu somente nas últimas duas décadas (BRIERE, 1997). Isso, de alguma forma, justifica a necessidade de que os estudos na área sejam desenvolvidos.

De modo especial, no Brasil, pode-se afirmar que o tema tornou-se mais relevante a partir da década de 1980, o que pôde ser confirmado pelos dados do presente trabalho. Cabe, no entanto, salientar que se usaram, a título de coleta de informações, apenas os resumos de manuscritos disponíveis on-line e, sabe-se que são poucas as revistas que possuem sua produção completa disponibilizada na Internet. Em síntese, embora a tendência seja afirmar categoricamente que houve aumento do número de artigos sobre estresse, há que se afirmar isso com cautela, uma vez que não foi avaliado o universo de periódicos nacionais.

Outro aspecto que merece destaque é que, embora a construção, validação e padronização de instrumentos não tenham sido abordadas com muita freqüência nos trabalhos consultados, os testes psicológicos foram utilizados como forma de coletar os dados. Espera-se com isso que haja avanço também no que se refere à construção de novos instrumentos. Ainda há que se salientar que apesar do avanço de pesquisas na área, especificamente na construção de testes, verifica-se que a quantidade de instrumentos aprovados pelo Conselho Federal de Psicologia, de fato, não é suficiente para responder a todas as especificidades do tema. 
Embora o presente trabalho não tenha permitido um amplo panorama das pesquisas no Brasil sobre estresse, até porque essa não era sua intenção, acreditase que os objetivos foram alcançados. Foi possível ter conhecimento de alguns elementos que podem servir de subsídios para pesquisas futuras, dentre eles, tipo de autoria, tipo de estudo, temas investigados, dentre outros. À guisa de consideração final, a verificação da situação do construto por meio de bases de dados on-line também possibilitará a identificação deste tipo de publicação científica.

\section{REFERÊNCIAS}

BARDIN, L. Análise de Conteúdo. São Paulo: Edições 70, 1971.

BEEHR, T. A. Research on occupational stress: an unfinished enterprise. Personnel Psychology, Greensboro, v. 51, p. 835-844, 1998.

BRIERE, J. Psychological assessment of adult posttraumatic states. Washington: American Psychological Association, 1997.

CADIZ, B. T. G. et al. "Burnout” profesional ¿Un problema nuevo? Reflexiones sobre el concepto y su evaluación. Revista de Psicología del Trabajo y de las Organizaciones, Madrid, v. 13, n. 1, p. 23-50, 1997.

CALAIS, S. L.; ANDRADE, L. M. B.; LIPP, M. E. N. Diferenças de sexo e escolaridade na manifestação de Stress em adultos jovens. Psicologia Reflexão e Crítica, Porto Alegre, v. 16, n. 2, p. 257-263, 2003.

CARLSON, N. R. Foundations of physiological psychology. Boston: Allyn and Bacon, 1995.

HAAN, N. The assessment of coping, defense and stress. In: GOLDBERGER, L.; BREZNITZ, S. (Org.). Handbook of stress: theoretical and clinical aspects. New York: Free Pass, 1982. p. 258-273.

HAMILTON, V. Cognition and stress: an information processing model. In: GOLDBERGER, L.; BREZNITZ, S. (Org). Handbook of Stress: theoretical and clinical aspects. London: Collier Macmillan, 1982. p. 105-120.

HINKLE Jr., L. E. Stress and disease the concept after 50 years. Social Science and Medicine, Leicester, v. 25, p. 561-566, 1987.

HOLROYD, K. A.; LAZARUS, R. S. Stress, coping, and somatic adaptation. In: GOLDBERGER, L.; BREZNITZ, S. (Org.). Handbook of Stress: theoretical and clinical aspects. New York: Free Pass, 1982. p. 21-35.

JAFFE, D. T. The healthy company: research paradigms for personal and organizational health. In: SAUTER, S. L.; MURPHY, L. R. (Org.). Organizational risk factors for job stress. Washington: American Psychological Association, 1995. p. 13-40. 
LIPP, M. E. N. Inventário de sintomas do stress para adulto. São Paulo: Casa do Psicólogo, 2000.

LIPP, M. E. N.; ARANTES, J. P.; BURITI, M. S. O estresse em escolares. Psicologia Escolar e Educacional, São Paulo, v. 6, n. 1, 2002, p. 51-56.

MAES, S.; VINGERHOETS, A.; HECK, G. van. The study of stress and disease: some developments and requirements. Social Science and Medicine, Leicester, v. 25, n. 6, p. 567-578, 1987.

MEIS, L.; LETA, J. O perfil da ciência brasileira. Rio de Janeiro: UFRJ, 1996.

MILLS, F. J. The endocrinology of stress. Aviation Space Environment Medicine, New York, v. 56, n. 7, p. 642-650, 1985.

MOTA, E. L. A.; FRANCO, A. L. S.; MOTTA, M. C. Migração, estresse e fatores psicossociais na determinação da saúde da criança. Psicologia Reflexão e Crítica, Porto Alegre, v. 12, n. 1, p. 119-132, 1999.

MUGNAINI, R.; CARVALHO, T.; CAMPANATTI-OSTIZ, H. Indicadores da produção científica: uma discussão conceitual. In: POBLACION, D. A.; WITTER, G. P.; SILVA, J. F. M. (Org.). Comunicação e Produção Cientifica. São Paulo: Angellara, 2006. p. 313-340

PEARLIN, L. I. The social contexts of stress. In: GOLDBERGER, L.; BREZNITZ, S. (Org.). Handbook of stress: theoretical and clinical aspects. London: Collier Macmillan, 1982. p. 1-6.

SELYE, H. The stress of life. New York: MacGraw-Hill, 1956.

. History and present status of the stress concept. In: GOLDBERGER, L.; BREZNITZ, S. (Org.). Handbook of stress: theoretical and clinical aspects. New York: Free Pass, 1982. p. 7-17.

WITTER, G. P. (Org.). A pesquisa educacional nas primeiras Reuniões da SBPC. In:__ Leitura: textos e pesquisas. Campinas: Alínea, 1979. p. 13-22.

. Produção científica: escalas de avaliação. In: POBLACION, D. A.; WITTER, G. P.; SILVA, J. F. M. (Org.). Comunicação e Produção Científica. São Paulo: Angellara, 2006. p. 261-285.

Recebido em: setembro de 2007

Aceito em: setembro de 2008 
